\title{
Active Epilepsy and Seizure Control in Adults — United States, 2013 and 2015
}

\author{
Niu Tian, MD, $\mathrm{PhD}^{1}$; Michael Boring, MS ${ }^{1,2}$; Rosemarie Kobau, MPH${ }^{1}$; Matthew M. Zack, MD ${ }^{1}$; Janet B. Croft, $\mathrm{PhD}^{1}$
}

Approximately 3 million American adults reported active epilepsy* in 2015 (1). Active epilepsy, especially when seizures are uncontrolled, poses substantial burdens because of somatic, neurologic, and mental health comorbidity; cognitive and physical dysfunction; side effects of antiseizure medications; higher injury and mortality rates; poorer quality of life; and increased financial cost (2). Thus, prompt diagnosis and seizure control (i.e., seizure-free in the 12 months preceding the survey) confers numerous clinical and social advantages to persons with active epilepsy. To obtain recent and reliable estimates of active epilepsy and seizure control status in the U.S. population, CDC analyzed aggregated data from the 2013 and the 2015 National Health Interview Surveys (NHISs). Overall, an annual estimated 2.6 million (1.1\%) U.S. adults self-reported having active epilepsy, $67 \%$ of whom had seen a neurologist or an epilepsy specialist in the past year, and $90 \%$ of whom reported taking epilepsy medication. Among those taking epilepsy medication, only $44 \%$ reported having their seizures controlled. A higher prevalence of active epilepsy and poorer seizure control were associated with low family income, unemployment, and being divorced, separated, or widowed. Use of epilepsy medication was higher among adults who saw an epilepsy specialist in the past year than among those who did not. Health care and public health should ensure that adults with uncontrolled seizures have appropriate care and self-management support in order to promote seizure control, improve health and social outcomes, and reduce health care costs.

NHIS is an annual, nationally representative household survey of the U.S. civilian, noninstitutionalized population. ${ }^{\dagger}$

\footnotetext{
*Among those reporting that a doctor or health professional had told them they had a seizure disorder or epilepsy, those who reported taking medication, having had one or more seizures in the past year, or both were considered to have active epilepsy.

${ }^{\dagger}$ https://www.cdc.gov/nchs/nhis/index.htm.
}

Epilepsy data were collected in the NHIS Sample Adult component, which includes one randomly selected adult aged $\geq 18$ years from each randomly selected household. In 2013, 34,557 adults (61.2\% final response rate) responded to the survey, and in 2015, 33,672 adults (55.2\% final response rate) responded. ${ }^{\S}$ Data for 2013 and 2015 were aggregated to provide more reliable estimates $(58.2 \%$ combined response rate). After excluding respondents with missing information on epilepsy history, 68,174 (99.9\%) respondents were included in the analysis.

$\overline{\$}$ https://www.cdc.gov/nchs/nhis/data-questionnaires-documentation.htm.

\section{INSIDE}

443 Protracted Outbreak of Salmonella Newport Infections Linked to Ground Beef: Possible Role of Dairy Cows - 21 States, 2016-2017

447 Assessment of Community Awareness and Practices Concerning Indoor Air Pollutants - Madison County, Alabama, June 2017

451 Suicidal Ideation and Attempts Among Students in Grades 8, 10, and 12 - Utah, 2015

455 Recommendations of the Advisory Committee on Immunization Practices for Use of a Hepatitis B Vaccine with a Novel Adjuvant

459 Notes from the Field: Surveillance for Candida auris Colombia, September 2016-May 2017

462 QuickStats

Continuing Education examination available at https://www.cdc.gov/mmwr/cme/conted_info.html\#weekly.

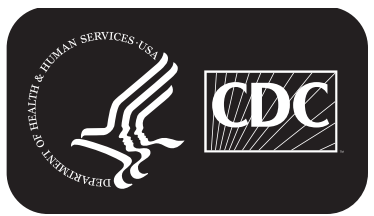

U.S. Department of Health and Human Services Centers for Disease Control and Prevention 
Adult respondents answered three questions about epilepsy to identify persons with active epilepsy and one question regarding specialty care. These case-ascertainment questions have been validated for use in community surveillance (3). Prevalence of active epilepsy and percentages of respondents with epilepsy who had seen a neurologist or epilepsy specialist in the past year, who were taking epilepsy medication, and whose seizures were controlled (i.e., had no seizures during the past year) among those taking epilepsy medication were estimated for each survey year, both survey years, overall, and by selected sociodemographic characteristics. The percentages of adults taking epilepsy medication and the distribution of seizure frequencies among those with active epilepsy by epilepsy specialty care were also estimated. Prevalences and percentages were age-standardized to the projected 2000 U.S. adult population by four age groups: 18-34, 35-54, 55-64, and $\geq 65$ years. Unless otherwise noted, the relative standard error of all estimates was $<30.0 \%$. Statistical software that accounted for the respondent sampling weights and the NHIS complex sample design was used for analysis. All reported differences between subgroups were statistically significant $(\mathrm{p}<0.05$ by two-tailed t-tests).

\footnotetext{
91) "Have you ever been told by a doctor or other health professional that you have a seizure disorder or epilepsy?" 2) "Are you currently taking any medicine to control your seizure disorder or epilepsy?” 3) "Think back to last year about the same time. About how many seizures of any type have you had in the past year?" and 4) "In the past year have you seen a neurologist or epilepsy specialist for your epilepsy or seizure disorder?"
}

\begin{abstract}
Summary
What is already known about this topic?

Approximately 3 million American adults have active epilepsy (doctor-diagnosed history of epilepsy, currently taking medication or having at least one seizure in the past year, or both). Uncontrolled seizures harm health, impair quality of life, and increase health care costs.

What is added by this report?

Although $90 \%$ of adults with active epilepsy were taking epilepsy medication, less than half (44\%) of those taking medications were seizure-free in the past year. Seizures were more common among persons with lower household income, the unemployed, and the divorced, separated, or widowed.

What are the implications for public health practice?

Health care and public health should ensure that adults with uncontrolled seizures have appropriate care and self-management support in order to promote seizure control, improve health and social outcomes, and reduce health care costs.
\end{abstract}

During 2013 and 2015, the annual prevalence of active epilepsy was $1.1 \%$ (approximately 2.6 million adults) and was significantly higher in 2015 (1.2\%) than in 2013 (0.9\%). The age-adjusted prevalence of active epilepsy was significantly higher among respondents who were non-Hispanic white (white) and non-Hispanic black (black); never married, divorced, separated, or widowed; had less than high school diploma; were unemployed, or living in lower-income families

The MMWR series of publications is published by the Center for Surveillance, Epidemiology, and Laboratory Services, Centers for Disease Control and Prevention (CDC), U.S. Department of Health and Human Services, Atlanta, GA 30329-4027.

Suggested citation: [Author names; first three, then et al., if more than six.] [Report title]. MMWR Morb Mortal Wkly Rep 2018;67:[inclusive page numbers].

\author{
Centers for Disease Control and Prevention \\ Robert R. Redfield, MD, Director \\ Anne Schuchat, MD, Principal Deputy Director \\ Leslie Dauphin, PhD, Acting Associate Director for Science \\ Joanne Cono, MD, ScM, Director, Office of Science Quality \\ Chesley L. Richards, MD, MPH, Deputy Director for Public Health Scientific Services \\ Michael F. Iademarco, MD, MPH, Director, Center for Surveillance, Epidemiology, and Laboratory Services
}

\title{
MMWR Editorial and Production Staff (Weekly)
}

Charlotte K. Kent, PhD, MPH, Acting Editor in Chief, Executive Editor Jacqueline Gindler, MD, Editor

Mary Dott, MD, MPH, Online Editor

Teresa F. Rutledge, Managing Editor

Douglas W. Weatherwax, Lead Technical Writer-Editor

Glenn Damon, Soumya Dunworth, PhD, Teresa M. Hood, MS, Technical Writer-Editors

\author{
Martha F. Boyd, Lead Visual Information Specialist \\ Maureen A. Leahy, Julia C. Martinroe, \\ Stephen R. Spriggs, Tong Yang, \\ Visual Information Specialists \\ Quang M. Doan, MBA, Phyllis H. King, \\ Paul D. Maitland, Terraye M. Starr, Moua Yang, \\ Information Technology Specialists
}

Timothy F. Jones, MD, Chairman

Matthew L. Boulton, MD, MPH

Virginia A. Caine, MD

Katherine Lyon Daniel, $\mathrm{PhD}$

Jonathan E. Fielding, MD, MPH, MBA

David W. Fleming, MD

\author{
MMWR Editorial Board \\ William E. Halperin, MD, DrPH, MPH \\ King K. Holmes, MD, PhD \\ Robin Ikeda, MD, MPH \\ Rima F. Khabbaz, MD \\ Phyllis Meadows, PhD, MSN, RN \\ Jewel Mullen, MD, MPH, MPA
}

\author{
Jeff Niederdeppe, $\mathrm{PhD}$ \\ Patricia Quinlisk, MD, MPH \\ Patrick L. Remington, MD, MPH \\ Carlos Roig, MS, MA \\ William L. Roper, MD, MPH \\ William Schaffner, MD
}


(e.g., families earning $<200 \%$ of federal poverty level [FPL]) than among other groups (Table).

The percentage of respondents with active epilepsy who had seen a neurologist or an epilepsy specialist in the past year was $67 \%$. This percentage was significantly higher among respondents aged 18-34 years, with at least some college education, or who lived in the Northeast than that among respondents aged $\geq 55$ years, who had less than a high school education, or who lived in other regions.

Ninety percent of respondents with active epilepsy took epilepsy medication, and this percentage did not significantly differ by sociodemographic characteristics. Among respondents taking epilepsy medication, $44 \%$ reported that their seizures were controlled in the past year. The prevalence of seizure control was significantly higher among adults aged $\geq 65$ years $(62.7 \%)$ than among those aged $35-54$ years $(36.9 \%)$; among persons who were married/cohabiting $(50.0 \%)$ than among those who were divorced, separated, or widowed $(31.5 \%)$; among persons who were employed (54.3\%) than among those who were unemployed (37.7\%); and among those with higher family incomes ( $\geq 200 \%$ of FPL; $55.3 \%$ ) than among those from lower income households ( $<200 \%$ of FPL; $33.2 \%$ ). By region, the prevalence of seizure control among respondents with epilepsy taking epilepsy medication who lived in the Northeast $(60.3 \%)$ was significantly higher than those who lived in the South (37.5\%).

TABLE. Number and age-adjusted* prevalence of active epilepsy, and percentages of adults who accessed specialty care, took epilepsy medications for seizure control, and were seizure-free with epilepsy medication in the past year among doctor-diagnosed active epilepsy, ${ }^{\dagger}$ by selected characteristics - National Health Interview Survey, United States, 2013 and 2015

\begin{tabular}{|c|c|c|c|c|c|c|c|c|c|c|c|c|}
\hline \multirow[b]{2}{*}{ Characteristic } & \multicolumn{3}{|c|}{ Adults with active epilepsy } & \multicolumn{3}{|c|}{$\begin{array}{l}\text { Seen a neurologist or } \\
\text { epilepsy specialist }\end{array}$} & \multicolumn{3}{|c|}{$\begin{array}{c}\text { Taking epilepsy medication to } \\
\text { control seizure }\end{array}$} & \multicolumn{3}{|c|}{$\begin{array}{l}\text { Seizure-free with } \\
\text { epilepsy medication }\end{array}$} \\
\hline & No. & $\begin{array}{c}\text { No. } \\
\text { (weighted) })^{\S}\end{array}$ & $\begin{array}{c}\text { Age- } \\
\text { adjusted \% } \\
(95 \% \mathrm{Cl})\end{array}$ & No. & $\begin{array}{l}\text { No. } \\
\text { (weighted)§ }\end{array}$ & $\begin{array}{l}\text { Age- } \\
\text { adjusted \% } \\
(95 \% \mathrm{Cl})\end{array}$ & No. & $\begin{array}{l}\text { No. } \\
\text { (weighted) }\end{array}$ & $\begin{array}{l}\text { Age- } \\
\text { adjusted \% } \\
(95 \% \mathrm{Cl})\end{array}$ & No. & $\begin{array}{c}\text { No. } \\
\text { (weighted)§ }\end{array}$ & $\begin{array}{c}\text { Age- } \\
\text { adjusted \% } \\
(95 \% \mathrm{Cl})\end{array}$ \\
\hline \multicolumn{13}{|l|}{ Survey year } \\
\hline 2013 & 367 & $2,254,000$ & $0.9(0.8-1.1)$ & 217 & $1,428,000$ & $\begin{array}{c}65.7 \\
(59.1-71.8)\end{array}$ & 305 & $1,948,000$ & $\begin{array}{c}86.3 \\
(81.1-90.3)\end{array}$ & 136 & 871,000 & $\begin{array}{c}45.3 \\
(37.3-53.6)\end{array}$ \\
\hline 2015 & 401 & $2,978,000$ & $1.2(1.1-1.4)$ & 255 & $2,032,000$ & $\begin{array}{c}68.3 \\
(62.1-74.0)\end{array}$ & 352 & $2,749,000$ & $\begin{array}{c}93.0 \\
(89.8-95.3)\end{array}$ & 152 & $1,184,000$ & $\begin{array}{c}42.4 \\
(35.0-50.2)\end{array}$ \\
\hline Total (crude) & 768 & $2,616,000$ & $1.1(1.0-1.2)$ & 472 & $1,730,000$ & $\begin{array}{c}66.2 \\
(61.6-70.5)\end{array}$ & 657 & $2,348,000$ & $\begin{array}{c}90.2 \\
(87.4-92.4)\end{array}$ & 288 & $1,028,000$ & $\begin{array}{c}44.1 \\
(38.7-49.7)\end{array}$ \\
\hline Total (age-adjusted) & 768 & $2,616,000$ & $1.1(1.0-1.2)$ & 472 & $1,730,000$ & $\begin{array}{c}67.0 \\
(62.6-71.2)\end{array}$ & 657 & $2,348,000$ & $\begin{array}{c}90.2 \\
(87.4-92.4)\end{array}$ & 288 & $1,028,000$ & $\begin{array}{c}43.7 \\
(38.1-49.5)\end{array}$ \\
\hline \multicolumn{13}{|l|}{ Sex } \\
\hline Men & 354 & $1,327,000$ & $1.1(1.0-1.3)$ & 224 & 919,000 & $\begin{array}{c}69.7 \\
(62.9-75.7)\end{array}$ & 313 & $1,221,000$ & $\begin{array}{c}92.0 \\
(87.5-95.0)\end{array}$ & 146 & 538,000 & $\begin{array}{c}43.7 \\
(36.1-51.5)\end{array}$ \\
\hline Women & 414 & $1,289,000$ & $1.0(0.9-1.2)$ & 248 & 811,000 & $\begin{array}{c}64.5 \\
(58.4-70.1)\end{array}$ & 344 & $1,128,000$ & $\begin{array}{c}88.3 \\
(84.2-91.4)\end{array}$ & 142 & 490,000 & $\begin{array}{c}43.5 \\
(35.7-51.6)\end{array}$ \\
\hline \multicolumn{13}{|l|}{ Age group (yrs) } \\
\hline $18-34$ & 165 & 803,000 & $1.1(0.9-1.4)$ & 116 & 615,000 & $\begin{array}{c}76.7 \\
(67.8-83.7)\end{array}$ & 136 & 721,000 & $\begin{array}{c}91.0 \\
(85.8-94.4)\end{array}$ & 54 & 303,000 & $\begin{array}{c}42.3 \\
(30.9-54.5)\end{array}$ \\
\hline $35-54$ & 280 & 867,000 & $1.0(0.9-1.2)$ & 175 & 585,000 & $\begin{array}{c}67.5 \\
(59.9-74.2)\end{array}$ & 235 & 768,000 & $\begin{array}{c}88.6 \\
(83.1-92.4)\end{array}$ & 80 & 280,000 & $\begin{array}{c}36.9 \\
(28.5-46.1)\end{array}$ \\
\hline $55-64$ & 165 & 540,000 & $1.4(1.1-1.6)$ & 95 & 305,000 & $\begin{array}{c}56.5 \\
(46.5-66.1)\end{array}$ & 144 & 483,000 & $\begin{array}{c}89.4 \\
(82.9-93.6)\end{array}$ & 63 & 209,000 & $\begin{array}{c}43.7 \\
(33.5-54.5)\end{array}$ \\
\hline$>65$ & 158 & 404,000 & $0.9(0.7-1.1)$ & 86 & 225,000 & $\begin{array}{c}55.6 \\
(45.0-65.7)\end{array}$ & 142 & 377,000 & $\begin{array}{c}93.1 \\
(88.0-96.1)\end{array}$ & 91 & 236,000 & $\begin{array}{c}62.7 \\
(50.7-73.3)\end{array}$ \\
\hline \multicolumn{13}{|l|}{ Race/Ethnicity } \\
\hline White, non-Hispanic & 507 & $1,857,000$ & $1.2(1.0-1.3)$ & 306 & $1,246,000$ & $\begin{array}{c}67.9 \\
(62.6-72.8)\end{array}$ & 440 & $1,692,000$ & $\begin{array}{c}91.4 \\
(88.0-93.9)\end{array}$ & 216 & 811,000 & $\begin{array}{c}47.5 \\
(40.4-54.6)\end{array}$ \\
\hline Black, non-Hispanic & 136 & 401,000 & $1.4(1.1-1.7)$ & 84 & 233,000 & $\begin{array}{c}62.8 \\
(50.6-73.7)\end{array}$ & 114 & 348,000 & $\begin{array}{c}88.8 \\
(81.0-93.6)\end{array}$ & 35 & 104,000 & $\begin{array}{c}32.3 \\
(21.5-45.3)\end{array}$ \\
\hline Other & 125 & 357,000 & $0.7(0.6-0.9)$ & 82 & 251,000 & $\begin{array}{c}70.5 \\
(59.4-79.6)\end{array}$ & 103 & 309,000 & $\begin{array}{c}86.5 \\
(78.8-91.8)\end{array}$ & 37 & 113,000 & $\begin{array}{c}37.3 \\
(26.7-49.3)\end{array}$ \\
\hline \multicolumn{13}{|l|}{ Marital status } \\
\hline Never married & 246 & 934,000 & $2.0(1.6-2.3)$ & 165 & 691,000 & $\begin{array}{c}71.0 \\
(62.5-78.2)\end{array}$ & 219 & 862,000 & $\begin{array}{c}92.7 \\
(87.6-95.8)\end{array}$ & 87 & 351,000 & $\begin{array}{c}44.9 \\
(35.3-55.0)\end{array}$ \\
\hline $\begin{array}{l}\text { Married/ } \\
\text { Cohabitating }\end{array}$ & 255 & $1,036,000$ & $0.7(0.6-0.8)$ & 156 & 667,000 & $\begin{array}{c}64.5 \\
(56.4-71.9)\end{array}$ & 218 & 932,000 & $\begin{array}{c}88.3 \\
(82.5-92.3)\end{array}$ & 106 & 441,000 & $\begin{array}{c}50.0 \\
(41.5-58.5)\end{array}$ \\
\hline $\begin{array}{l}\text { Divorced/ } \\
\text { Separated/ } \\
\text { Widowed }\end{array}$ & 266 & 641,000 & $1.7(1.2-2.3)$ & 150 & 367,000 & $\begin{array}{c}63.9 \\
(53.4-73.2)\end{array}$ & 219 & 550,000 & $\begin{array}{c}85.8 \\
(76.6-91.8)\end{array}$ & 95 & 236,000 & $\begin{array}{c}31.5 \\
(25.3-38.4)\end{array}$ \\
\hline
\end{tabular}

See table footnotes on the next page. 
TABLE. (Continued) Number and age-adjusted* prevalence of active epilepsy, and percentages of adults who accessed specialty care, took epilepsy medications for seizure control, and were seizure-free with epilepsy medication in the past year among doctor-diagnosed active epilepsy, ${ }^{\dagger}$ by selected characteristics - National Health Interview Survey, United States, 2013 and 2015

\begin{tabular}{|c|c|c|c|c|c|c|c|c|c|c|c|c|}
\hline \multirow[b]{2}{*}{ Characteristic } & \multicolumn{3}{|c|}{ Adults with active epilepsy } & \multicolumn{3}{|c|}{$\begin{array}{c}\text { Seen a neurologist or epilepsy } \\
\text { specialist }\end{array}$} & \multicolumn{3}{|c|}{$\begin{array}{l}\text { Taking epilepsy medication to } \\
\text { control seizure }\end{array}$} & \multicolumn{3}{|c|}{$\begin{array}{l}\text { Seizure-free with epilepsy } \\
\text { medication }\end{array}$} \\
\hline & No. & $\begin{array}{c}\text { No. } \\
\text { (weighted) }\end{array}$ & $\begin{array}{c}\text { Age- } \\
\text { adjusted \% } \\
(95 \% \mathrm{Cl})\end{array}$ & No. & $\begin{array}{c}\text { No. } \\
\text { (weighted) }^{\S}\end{array}$ & $\begin{array}{c}\text { Age- } \\
\text { adjusted \% } \\
(95 \% \mathrm{Cl})\end{array}$ & No. & $\begin{array}{c}\text { No. } \\
\text { (weighted) }\end{array}$ & $\begin{array}{c}\text { Age- } \\
\text { adjusted \% } \\
(95 \% \mathrm{Cl})\end{array}$ & No. & $\begin{array}{c}\text { No. } \\
\text { (weighted) }\end{array}$ & $\begin{array}{c}\text { Age- } \\
\text { adjusted \% } \\
(95 \% \mathrm{Cl})\end{array}$ \\
\hline \multicolumn{13}{|l|}{ Education level } \\
\hline Less than HS & 194 & 564,000 & $1.8(1.5-2.2)$ & 104 & 310,000 & $\begin{array}{c}58.7 \\
(48.9-67.9)\end{array}$ & 164 & 498,000 & $\begin{array}{c}89.4 \\
(82.9-93.6)\end{array}$ & 63 & 192,000 & $\begin{array}{c}39.0 \\
(28.9-50.2)\end{array}$ \\
\hline HS diploma or GED & 216 & 803,000 & $1.3(1.1-1.6)$ & 131 & 496,000 & $\begin{array}{c}62.4 \\
(53.5-70.5)\end{array}$ & 190 & 733,000 & $\begin{array}{c}92.5 \\
(88.0-95.4)\end{array}$ & 76 & 283,000 & $\begin{array}{c}38.9 \\
(30.2-48.4)\end{array}$ \\
\hline Some college & 348 & $1,203,000$ & $0.8(0.7-0.9)$ & 231 & 896,000 & $\begin{array}{c}74.2 \\
(67.8-79.8)\end{array}$ & 294 & $1,074,000$ & $\begin{array}{c}89.0 \\
(84.3-92.4)\end{array}$ & 144 & 533,000 & $\begin{array}{c}49.4 \\
(41.3-57.6)\end{array}$ \\
\hline \multicolumn{13}{|l|}{ Current employment } \\
\hline Yes & 215 & 783,000 & $0.5(0.4-0.6)$ & 129 & 538,000 & $\begin{array}{c}67.9 \\
(59.4-75.5)\end{array}$ & 183 & 709,000 & $\begin{array}{c}89.6 \\
(83.8-93.5)\end{array}$ & 104 & 376,000 & $\begin{array}{c}54.3 \\
(44.1-64.1)\end{array}$ \\
\hline No & 553 & $1,833,000$ & $2.5(2.2-2.8)$ & 343 & $1,192,000$ & $\begin{array}{c}67.9 \\
(62.6-72.7)\end{array}$ & 474 & $1,639,000$ & $\begin{array}{c}90.1 \\
(86.7-92.8)\end{array}$ & 184 & 652,000 & $\begin{array}{c}37.7 \\
(31.0-45.1)\end{array}$ \\
\hline \multicolumn{13}{|l|}{ Poverty status** } \\
\hline$<200 \%$ of $\mathrm{FPL}$ & 481 & $1,383,000$ & $1.9(1.6-2.1)$ & 284 & 864,000 & $\begin{array}{c}64.6 \\
(58.4-70.4)\end{array}$ & 402 & $1,222,000$ & $\begin{array}{c}88.2 \\
(84.0-91.4)\end{array}$ & 143 & 399,000 & $\begin{array}{c}33.2 \\
(26.6-40.4)\end{array}$ \\
\hline$\geq 200 \%$ of $F P L$ & 287 & $1,233,000$ & $0.8(0.6-0.9)$ & 188 & 866,000 & $\begin{array}{c}70.6 \\
(63.4-76.9)\end{array}$ & 255 & $1,126,000$ & $\begin{array}{c}92.1 \\
(87.9-94.9)\end{array}$ & 145 & 628,000 & $\begin{array}{c}55.3 \\
(46.7-63.6)\end{array}$ \\
\hline \multicolumn{13}{|l|}{ Region $^{\dagger \dagger}$} \\
\hline Northeast & 112 & 390,000 & $0.9(0.7-1.2)$ & 82 & 316,000 & $\begin{array}{c}84.3 \\
(74.9-90.7)\end{array}$ & 97 & 358,000 & $\begin{array}{c}89.5 \\
(79.7-94.9)\end{array}$ & 46 & 209,000 & $\begin{array}{c}60.3 \\
(47.1-72.1)\end{array}$ \\
\hline Midwest & 157 & 549,000 & $1.0(0.8-1.2)$ & 94 & 349,000 & $\begin{array}{c}63.9 \\
(54.8-72.2)\end{array}$ & 136 & 491,000 & $\begin{array}{c}90.1 \\
(83.3-94.4)\end{array}$ & 67 & 233,000 & $\begin{array}{c}47.9 \\
(36.5-59.5)\end{array}$ \\
\hline South & 291 & $1,096,000$ & $1.2(1.1-1.4)$ & 177 & 706,000 & $\begin{array}{c}66.7 \\
(59.9-72.9)\end{array}$ & 251 & 977,000 & $\begin{array}{c}90.3 \\
(85.2-93.7)\end{array}$ & 98 & 374,000 & $\begin{array}{c}37.5 \\
(29.3-46.3)\end{array}$ \\
\hline West & 208 & 580,000 & $1.0(0.8-1.3)$ & 119 & 359,000 & $\begin{array}{c}61.5 \\
(51.8-70.4)\end{array}$ & 173 & 522,000 & $\begin{array}{c}89.3 \\
(84.0-93.0)\end{array}$ & 77 & 212,000 & $\begin{array}{c}41.4 \\
(31.0-52.5)\end{array}$ \\
\hline
\end{tabular}

Abbreviations: $\mathrm{Cl}=$ confidence interval; $\mathrm{GED}=$ General Educational Development; $\mathrm{HS}$ = high school; $\mathrm{FPL}=$ federal poverty level.

${ }^{*}$ Age-adjusted to the 2000 U.S. projected population, aged $\geq 18$ years, using four age groups: $18-34,35-54,55-64$, and $\geq 65$ years. All prevalence estimates are ageadjusted except those for age groups, and overall (crude).

† Doctor-diagnosed active epilepsy was defined as having a diagnosis of epilepsy and either taking medication or having had one or more seizures in the past year, or both.

$\S$ Annualized and weighted number rounded to 1,000 s.

" Other race/ethnicity includes non-Hispanic American Indian and Alaska Native only; non-Hispanic Asian only; non-Hispanic Native Hawaiian and Pacific Islander only; and non-Hispanic multiple race.

** Poverty status was defined as the ratio of family income to FPL.

${ }^{\dagger+}$ Northeast region (Connecticut, Maine, Massachusetts, New Hampshire, New Jersey, New York, Pennsylvania, Rhode Island, Vermont); Midwest region (Illinois, Indiana, lowa, Kansas, Michigan, Minnesota, Missouri, Nebraska, North Dakota, Ohio, South Dakota, Wisconsin); South region (Alabama, Arkansas, Delaware, District of Columbia, Florida, Georgia, Kentucky, Louisiana, Mississippi, Maryland, North Carolina, Oklahoma, South Carolina, Tennessee, Texas, Virginia, West Virginia); West region (Alaska, Arizona, California, Colorado, Hawaii, Idaho, Montana, Nevada, New Mexico, Oregon, Utah, Washington, Wyoming).

Among adults with active epilepsy, the age-adjusted prevalence of taking epilepsy medication was higher among those who saw an epilepsy specialist in the past year (95.4\%) than among those who did not (78.1\%); however, seizure frequency among those with active epilepsy did not differ significantly between those who did and did not see a specialist in the past year (Figure).

\section{Discussion}

The number of adults reporting that they have active epilepsy has significantly increased from 2010 (2.3 million) (4) to 2015 ( 3 million), with about 724,000 more cases identified from 2013 to 2015 . In 2010 , just over half $(52.8 \%)$ of adults with active epilepsy saw a neurologist or epilepsy specialist (4). This study found that approximately two thirds $(65.7 \%$ in 2013 and $68.3 \%$ in 2015) of adults with epilepsy saw a specialist. Most (90\%) respondents with active epilepsy were taking epilepsy medication. Epilepsy medication use, but not reduced seizure frequency, was more common among those who had seen an epilepsy specialist; however, only $44 \%$ of respondents who took epilepsy medication had their seizures controlled in the past year. These results suggest that apart from the improvement associated with prompt diagnosis and treatment, other factors that might affect seizure control need to be addressed. The finding that blacks and respondents with less education and lower income had higher prevalences of active epilepsy is 
FIGURE. Age-standardized prevalence of epilepsy medication use and seizure frequency among adults with active epilepsy, by receipt of specialty care in the past year — National Health Interview Survey, United States, 2013 and 2015

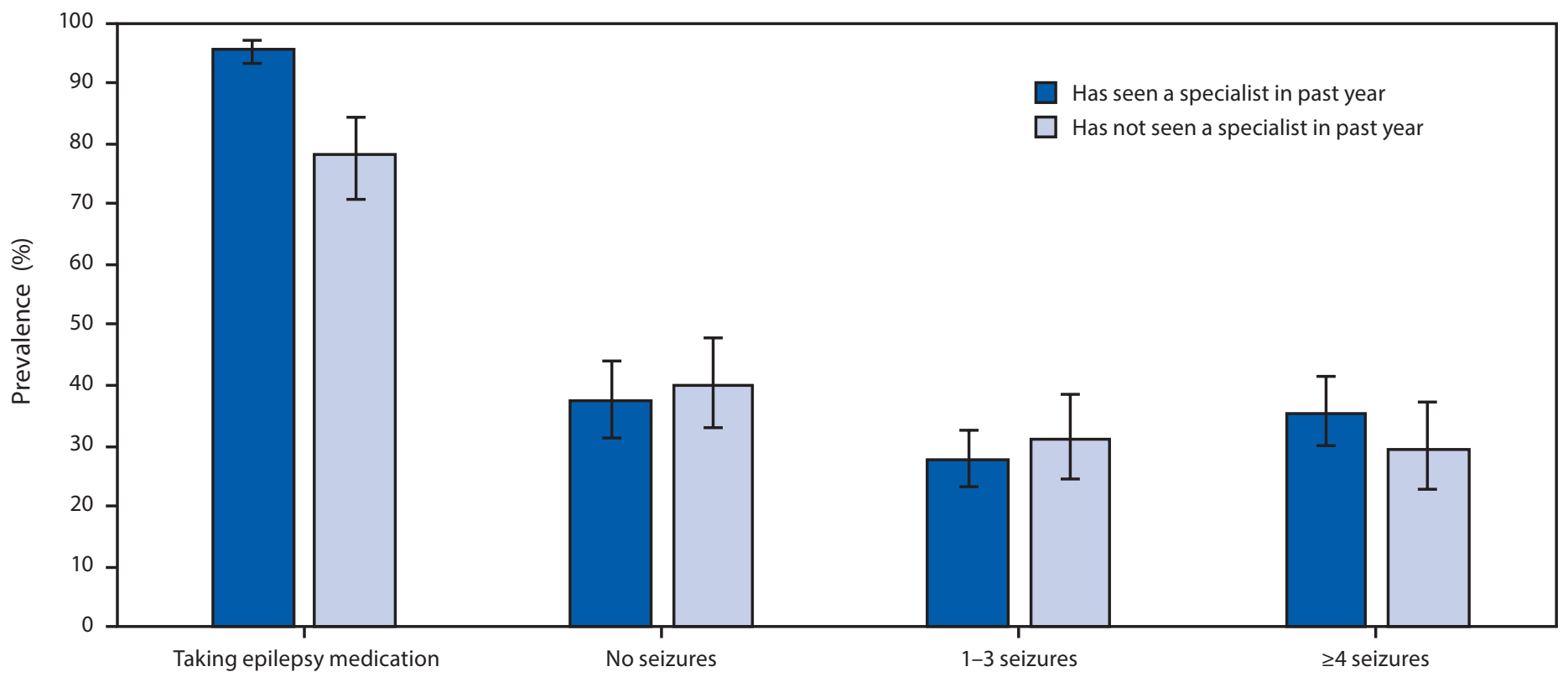

consistent with previous reports $(4,5)$. This study also found that poor seizure control was associated with low income, unemployment, and being divorced, separated, or widowed. Socioeconomic disadvantage among adults with active epilepsy might preclude their accessing health care including specialty care (because of barriers such as cost and transportation) (2), thus affecting seizure control. More importantly, socioeconomic disadvantage (e.g., less education) and social isolation (e.g., lack of social support associated with being divorced, separated, or widowed) (2) might lead to nonadherence to epilepsy medication $(\sigma)$, an important clinical factor that significantly hinders seizure control $(6,7)$.

Among adults taking epilepsy medication, those aged $\geq 65$ years had better seizure control than among younger adults aged 35-54 years. This finding is also consistent with a previous report (8). The apparent better response to epilepsy medication in older adults might be attributable to differences in seizure etiology, drug pharmacokinetics, or better adherence to prescribed antiseizure medication regimens, possibly because of their experience with other chronic conditions or better access to care, including Medicare prescription drug coverage.

Only $44 \%$ of respondents with active epilepsy on epilepsy medication in this study were seizure-free in the past year. According to the Institute of Medicine, about $70 \%$ of all patients with epilepsy might become seizure-free under appropriate epilepsy treatment (2). To optimize seizure control, clinicians' decisions to treat epilepsy should be based on individualized assessments of both disease-based (e.g., age of disease onset, seizure etiology, type, and comorbid conditions) and treatment-based factors (e.g., adherence to antiepileptic drugs), as well as patients' personal characteristics, preferences, and their social context $(6,7,9)$. Improving access to care, providing social support and epilepsy self-management education to improve medication adherence, and encouraging other selfmanagement behaviors such as avoiding seizure triggers (e.g., sleep deprivation, stress, flashing lights, and alcohol or drug use) might also improve seizure control (10).

The findings in this report are subject to at least five limitations. First, estimates of epilepsy prevalence are based on self-reported data and are subject to error; however, because previous studies have validated the NHIS epilepsy questions, this bias is expected to be small (3). Second, active epilepsy might be overestimated because of the mistaken reporting of other nonepileptic seizures (5) or underestimated because of respondents' reluctance to disclose epilepsy (2), as well as by the exclusion of institutionalized adults (e.g., adults in long-term care facilities and incarcerated persons) from NHIS. Third, these surveys did not objectively measure medication adherence or seizure frequency. Fourth, although respondent survey weights were adjusted to the U.S. population, the potential for nonresponse bias cannot be eliminated, given the low overall response rate $(58.2 \%)$. Finally, the lack of differences in seizure frequency by seeing a specialist could be confounded by epilepsy severity and other untreated comorbidity such as mood disorder. However, no data regarding epilepsy severity is collected on NHIS

These findings highlight both the substantial burden of uncontrolled seizures in adults with epilepsy and the persistent 
sociodemographic and socioeconomic disparities in active epilepsy prevalence, access to neurologic specialty care, and seizure control. Health care and public health should ensure that adults with uncontrolled seizures have appropriate care and self-management support in order to promote seizure control, improve health and social outcomes, and reduce health care costs.

\section{Conflict of Interest}

No conflicts of interest were reported.

${ }^{1}$ Division of Population Health, National Center for Chronic Disease Prevention and Health Promotion, CDC; ${ }^{2}$ Cutting Edge Technologies and Solutions (Cetechs), Mesa, Arizona.

Corresponding author: Niu Tian, NTian@cdc.gov, 770-488-5679.

\section{References}

1. Zack MM, Kobau R. National and state estimates of the numbers of adults and children with active epilepsy-United States, 2015. MMWR Morb Mortal Wkly Rep 2017;66:821-5. https://doi.org/10.15585/ mmwr.mm6631a1

2. Institute of Medicine. Epilepsy across the spectrum: promoting health and understanding. Washington, DC: The National Academy Press; 2012. https://www.nap.edu/read/13379/chapter/3\#25
3. Brooks DR, Avetisyan R, Jarrett KM, et al. Validation of self-reported epilepsy for purposes of community surveillance. Epilepsy Behav 2012;23:57-63. https://doi.org/10.1016/j.yebeh.2011.11.002

4. CDC. Epilepsy in adults and access to care-United States, 2010. MMWR Morb Mortal Wkly Rep 2012;61:909-13.

5. Kroner BL, Fahimi M, Kenyon A, Thurman DJ, Gaillard WD. Racial and socioeconomic disparities in epilepsy in the District of Columbia. Epilepsy Res 2013;103:279-87. https://doi.org/10.1016/j. eplepsyres.2012.07.005

6. Szaflarski M. Social determinants of health in epilepsy. Epilepsy Behav 2014;41:283-9. https://doi.org/10.1016/j.yebeh.2014.06.013

7. Garnett WR. Antiepileptic drug treatment: outcomes and adherence. Pharmacotherapy 2000;20:191S-9S. https://doi.org/10.1592/ phco.20.12.191S.35250

8. Stefan H, May TW, Pfäfflin M, et al. Epilepsy in the elderly: comparing clinical characteristics with younger patients. Acta Neurol Scand 2014;129:283-93. https://doi.org/10.1111/ane.12218

9. Manjunath R, Davis KL, Candrilli SD, Ettinger AB. Association of antiepileptic drug nonadherence with risk of seizures in adults with epilepsy. Epilepsy Behav 2009;14:372-8. https://doi.org/10.1016/j. yebeh.2008.12.006

10. Schachter SC. Seizure disorders. Med Clin North Am 2009;93:343-51, viii. https://doi.org/10.1016/j.mcna.2008.10.001 